\title{
Auch Held(innen) haben kranke Beine - eine Nachlese
}

Dass es Bielefeld sehr wohl gibt, hat uns die 60. Jahrestagung der Deutschen Gesellschaft für Phlebologie, die vom 26.29.9.2018 in der Stadthalle in Bielefeld stattfand, bewiesen.

Mit gut 1.000 Teilnehmern - 175 Teilnehmern am Crashkurs und Update Phlebologie schon am Vorkongresstag - ausgebuchten Hands-On-Workshops, einem rappelvollen Weiterbildungsforum und sehr gut besuchten Firmensymposien war die Jahrestagung eine rundum gelungene Veranstaltung.

Der Crash-Kurs und das Phlebo-Update machten den Auftakt in bewährter Form, organisiert von Frau PD Dr. Pannier und Frau Prof. Dr. Reich-Schupke. Mit 175 Teilnehmern bereits am „Vorkongresstag“ konnte der aktuelle Wissensstand der Phlebologie präsentiert und zur Diskussion gestellt werden. Erstmals wurde das Phlebo-Startup durchgeführt, dass sich an Medizinstudenten richtet. Die Referenten konnten interessierten angehenden Medizinern aus benachbarten Universitäten Grundlagen der phlebologischen Diagnostik und Therapie vermitteln. Ermöglicht wurde dieses neue Ausbildungsformat von der Fa. Sigvaris.

Schon die Auftaktveranstaltung zeigte sehr klar, wie sich die „Deutsche Phlebologie“ in den letzten 20 Jahren sowohl wissenschaftlich als auch von der Struktur der Gesellschaft her verändert hat. Viele junge Kolleginnen und Kollegen besuchten erstmals die Jahrestagung der Deutschen Gesellschaft für Phlebologie, ein schönes Indiz dafür, dass die gezielte Nachwuchsarbeit der Gesellschaft Früchte trägt. Diese positive Entwicklung drückt sich auch in der stetig steigenden Mitgliederzahl unserer Gesellschaft aus.
Im Anschluss erfreute uns Prof. Joachim Thalmann von der Hochschule für Musik in Detmold mit einem wunderbaren Vortrag zum Thema „Feldforschung einmal anders: Wenn Fans singen - Betrachtungen zum musikalischen Geschehen in den Südkurven“. Musikalisch untermalt wurde dieses spannende Thema von einem klassischen Ensemble mit Akkordeon und Klarinette und passend zu den Fangesängen mit der Blaskapelle Humfeld.

Das in den vergangenen Jahren bewährte Konzept des „aktiven wissenschaftlichen Beirats" hat sich auch in diesem Jahr wieder bewährt. Die Organisation einzelner wissenschaftlicher Sitzungen in den jeweiligen Spezialgebieten der Vorsitzenden hat dazu beigetragen, dass ein breit gefächertes Programm gestaltet werden konnte. Mal waren die Sitzungen von Kurzreferaten geprägt, die gegeneinander bewusst provozierend die Diskussionen herausforderten, mal waren Sitzungen von Grundsatzreferaten gekennzeichnet, die den wissenschaftlich aktuellen Status quo eines Themas präsentierten.

Wieder eingeführt wurden am Donnerstag und Freitagvormittag Plenarsitzungen mit besonders spannenden Themen, gekennzeichnet von überdurchschnittlicher Akzeptanz.

Prof. Peter Mortimer wurde im Rahmen der Ratschow-Sitzung die „Ratschow-Medaille“ verliehen. Sein Festvortrag zum Thema „Go with the (lymph) flow“ gehörte zu den Highlights der Tagung.

Die Ratschow-Gedächtnismedaille wird im Jahresrhythmus seit 1969 vom CURATORIUM ANGIOLOGIAE INTERNATIONALIS an besonders verdiente wissenschaftliche Per- sönlichkeiten aus dem Bereich Gefäßmedizin und diese tangierenden Disziplinen verliehen.

Das Weiterbildungsforum am Donnerstagvormittag war sehr gut besucht und bot den ,jungen Phlebologen“ die Möglichkeit, einen ersten Einblick in phlebologische Diagnostik und Therapie zu erhalten. Darüber hinaus standen mit den Vorsitzenden und Referenten kompetente Diskussionspartner zur Verfügung.

An allen drei Kongresstagen waren die „ask the experts"-Sitzungen früh um 8 Uhr gut besucht. Die Möglichkeit, Experten zu allen Themen, die Phlebologen in der Praxis beschäftigen, persönlich zu befragen und vor allem über eigene Erfahrungen und Fälle zu diskutieren, wurde gut angenommen.

Darüber hinaus wurde den täglichen Problemen des Praxisalltages reichlich Platz eingeräumt, so gab es wissenschaftliche Sitzungen zum Themenfeld Kompression, die Sklerosierungstherapie wurde behandelt und der große Raum der operativen und endoluminalen Therapie fand in zahlreichen Sitzungen Berücksichtigung. Venenerhaltende Therapiekonzepte bei der Stammvarikose konnten erläutert werden, ebenso wie die Thrombosetherapie. In zwei Sitzungen „Der interessante Fall“ wurden Situationen aus dem Praxis- und Klinikalltag geschildert und ausführlich diskutiert.

Dies ist nur ein kleiner Abriss der Inhalte der 60 . Jahrestagung. Neben den angebotenen wissenschaftlichen Inhalten lag ein Schwerpunkt in der Weiterbildung: Weiterbildungsforum, Vertiefungskurs zum Phlebologieassistenten, Hands-On-Kurse und zertifizierte Pflegefachtagung - um nur einige Aspekte zu nennen. 


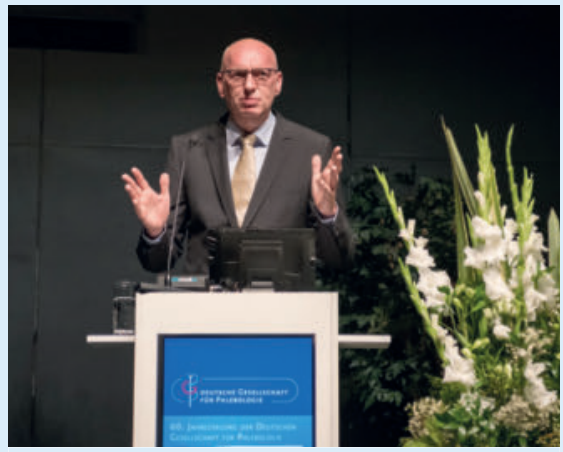

A Abb. 1 PD Dr. Helger Stege, Kongresspräsident der 60. Jahrestagung der DGP in Bielefeld

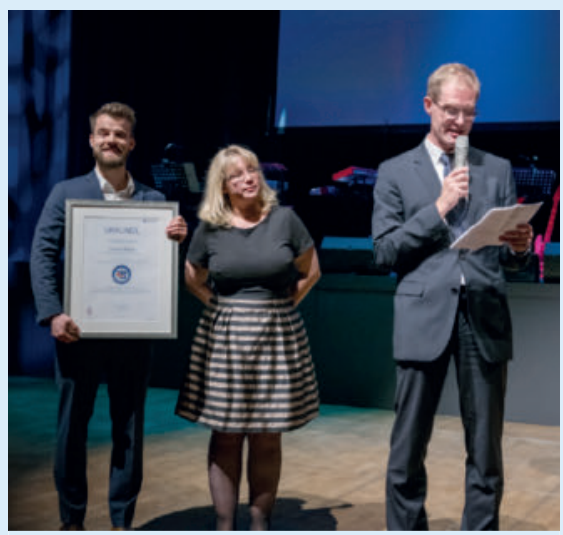

Abb. 4 Verleihung des BauerfeindDoktoranden-Preises: Preisträger Danie Rabe, Nadine Rüdiger (Bauerfeind AG), Prof. Stücker (v.l.n.r.)

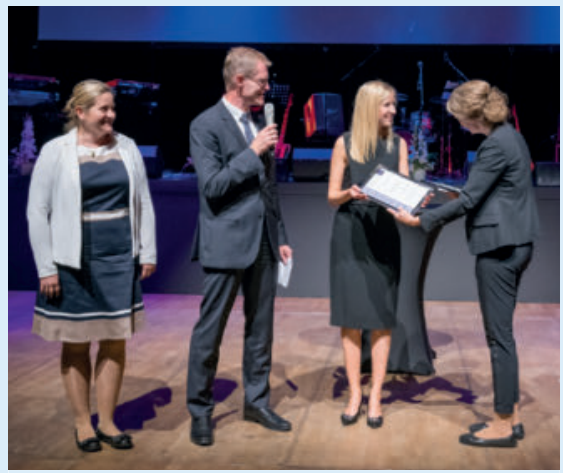

Abb. 7 Verleihung des Sigvaris Förderpreis Phlebologie: Preisträgerin Eva Cyrek, Prof. Stücker, Preisträgerin Jana Zion, Carola Töpfer (Sigvaris GmbH)

45 ausstellende Firmen konnten die Innovationen in der fachgebundenen Medizintechnik und der Pharmakologie begleiten und so unseren Besuchern auch auf diesem Gebiet aktuelles Wissen vermitteln. Durch die sogenannte Phleboarena, eine offene Vor-

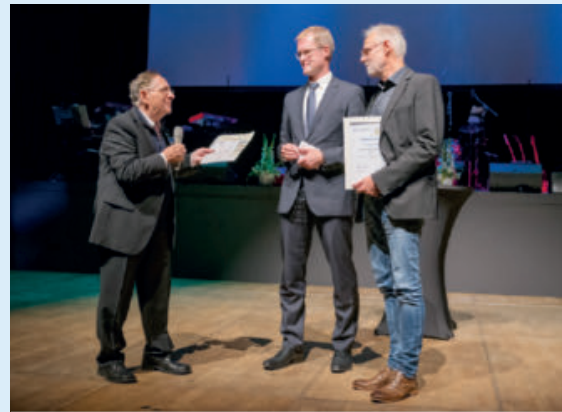

- Abb. 2 Vergabe des des VaradyVortragspreises an Dr. Meyer-Berendes (Prof. Varady, Prof. Stücker, Dr. MeyerBerendes) (v.I.n.r.)

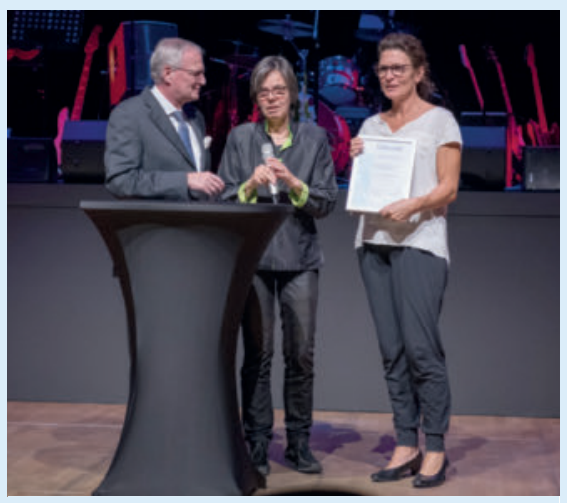

- Abb. 5 Der Thieme-Kasuistikpreis ging an Dr. Toni Silber, Tübingen. Dr. Thomas Noppeney und Prof. Birgit Kahle übergeben die Urkunde stellvertretend an Prof. Strölin, Tübingen (v.l.n.r.)

tragsfläche in der Industrieausstellung, gelang es, während der Pausen und darüber hinaus die Teilnehmer in die Ausstellungshalle zu ziehen und aktive Begegnungen zu ermöglichen.

In Bielefeld waren sowohl in der Stadthalle als auch beim Rahmenprogramm die Wege kurz, was intensive Dialoge und eine familiäre Atmosphäre bedeutete.

Wie immer stellte der Kongress die Plattform für Ehrungen und Preisverleihungen dar.

- Erstmals vergeben wurde der von Prof. Zoltan Varady gespendete Varady-Vortragspreis. Prof. Varady stiftet in Kooperation mit der Deutschen Gesellschaft für Phlebologie einen Wissenschaftspreis im Rahmen unserer Jahrestagung. Jeweils der beste Vortrag im Bereich der Minichirurgie oder Mikrochirurgie auf der Jahrestagung der Deutschen Gesellschaft für Phlebologie soll mit einem

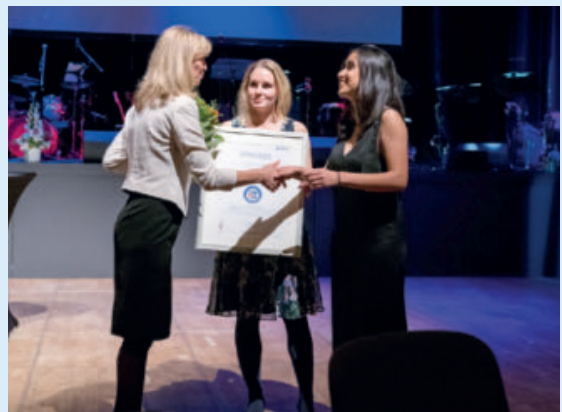

Abb. 3 Verleihung des BauerfeindDoktoranden-Preises: Dr. Mark, Bauerfeind AG (links) und Preisträgerin Julia Götz (rechts)

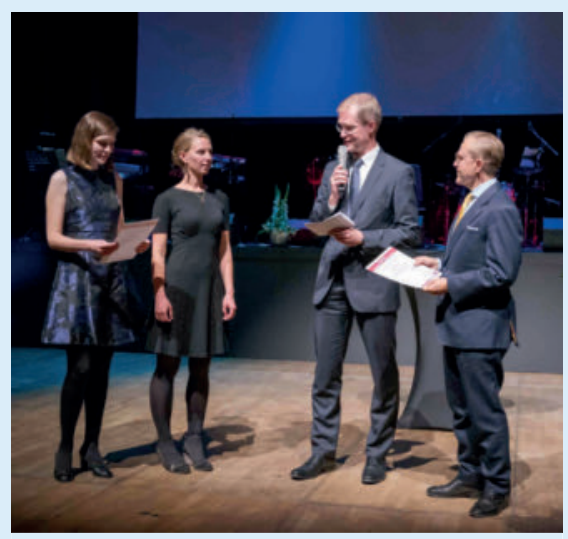

-Abb. 6 Kreussler-Stipendien-Preisträger 2018: Kira Süssmuth (Preisträgerin), Elena Sagolla (Preisträgerin), Prof. Stücker, Dr. Travers, Kreussler Pharma (v.I.n.r.)

Vortragspreis von $€ 1.000,00$ prämiert werden. In diesem Jahre geht der Preis an Dr. Meyer-Berendes aus Damme.

- Sigvaris-Förderpreis Phlebologie Preisträger 2018:

- Jana Ziob, Bonn

- Eva Cyrek, Essen

- Bauerfeind-Doktorandenprogramm Preisträger 2018:

- Daniel Rabe, Bonn, für seine Arbeit zum Thema: Aktuelle Behandlung und Kompressionsstrategien nach proximaler tiefer Beinvenenthrombose. - Ergebnisse einer internationalen Umfrage

- Julia Götz, Greifswald, für Ihre Arbeit zum Thema: Tragekomfort und Veränderung des transepidermalen Wasserverlustes bei knielangen elastischen Kompressionsstrümpfen mit einem Anpressdruck von 18-21 mm $\mathrm{Hg}$ im Vergleich zu 23-32 mm Hg 
bei älteren Menschen nach einem eintägigen Tragetest

- Thieme-Kasuistikpreis:

- Dr. Toni Silber, Tübingen, für seine Arbeit zum Thema: Fulminanter Verlauf eines Trousseau-Syndroms. Phlebologie 2018; 47: 133-136

- Kreussler-Stipendien - Preisträger 2018:

- Dr. Kira Süssmuth, Universitätshautklinik Münster: „Antikoagulation bei der Sklerotherapie?“

- Elena Sagolla, Venenzentrum der Dermatologischen und Gefäßchirur- gischen Kliniken, Ruhr-Universität Bochum: „Morphologie und Symptomatik einer unbehandelten Besenreiservarikose“

- Medi-Reisestipendien: 15 Preise à 500,00 Euro

Zum Abschluss noch der herzliche Dank an alle, die zum Gelingen der 60. Jahrestagung der DGP beigetragen haben - dem wissenschaftlichen Beirat, den aktiven Referenten und Vorsitzenden, der unterstützenden Industrie, ohne die eine solche Veranstaltung nicht möglich wäre, den Musikern des Rah- menprogramms, den vielen hilfsbereiten und freundlichen Hostessen, der Technik, der Kongressorganisation und dem zuarbeitenden Service, und vor allem dem Kongresspräsidenten PD Dr. Helger Stege.

Die nächste Jahrestagung findet 2019 in Münster statt. Vom 18.-21.9.2019 ist Prof. Görge unser Gastgeber in der Halle Münsterland. 